\title{
Stability of macroscopic traffic flow modeling through wavefront expansion
}

\author{
Jingang $\mathrm{Yi}^{\mathrm{a}, 1}$, Hao Lin ${ }^{\mathrm{a}, 2}$, Luis Alvarez ${ }^{\mathrm{b}}$, Roberto Horowitz ${ }^{\mathrm{a}, *}$ \\ a Department of Mechanical Engineering, University of California, Berkeley, CA 94720-1742, USA \\ b Instituto de Ingeniería Universidad Nacional Autónoma de México, 04510 Coyoacán DF, Mexico
}

Received 6 November 2001; received in revised form 12 September 2002; accepted 16 September 2002

\begin{abstract}
In this paper, second-order macroscopic vehicle traffic flow models are discussed from the perspective of their capability to reproduce stable and unstable traffic flow behaviors observed in real traffic. To achieve this goal, a nonlinear traffic flow stability criterion is derived using a wavefront expansion technique. Qualitative relationships between traffic flow stability and model parameters are derived for an entire class of second-order macroscopic traffic flow models. The stability criterion is illustrated by numerical results using the CLAWPACK package for the well-known Payne-Whitham (PW) model. The newly derived stability results are consistent with previously reported results obtained using both microscopic models and approximate linearization methods. Moreover, the stability criteria derived in this paper can provide more refined information regarding the propagation of traffic flow perturbations and shock waves in secondorder models than previously established methodologies.

(C) 2003 Elsevier Science Ltd. All rights reserved.
\end{abstract}

Keywords: Traffic stability; Macroscopic traffic models; Wavefront expansion

\footnotetext{
${ }^{*}$ Corresponding author. Tel.: +1-510-642-4675; fax: +1-510-643-5599.

E-mail addresses: jgyi@gandhi.me.berkeley.edu (J. Yi), lin@ctr.stanford.edu (H. Lin), alvar@pumas.iingen. unam.mx (L. Alvarez), horowitz@me.berkeley.edu (R. Horowitz).

${ }^{1}$ Currently with Lam Research Corporation, Fremont, CA 94538, USA.

${ }^{2}$ Currently a post-doctoral research fellow at Stanford University.
} 


\section{Introduction}

Modeling of traffic flow has been a key tool to simulate or predict the behavior of transportation systems. An important question regarding the applicability of these models lies in their capability to replicate how traffic congestions, an undesirable phenomenon that is observed in real traffic, occur when disturbances are present in transportation systems. In order to answer the stability question for a particular model, it is necessary to establish what are the critical model parameters or traffic flow conditions that lead, on one hand, to a stable response to perturbations or disturbances, or, on the other hand, to a unstable response and therefore to a possible congestion. There is a large body of research that deals with the stability of traffic flow. Holland (1998) discussed traffic stability for various well-known microscopic traffic flow models, i.e. car following models. Whitham (1974) presented a stability criterion for a well-known second-order macroscopic traffic flow model, known as the Payne-Whitham (PW) model. Swaroop and Rajagopal (1999) introduced a formal definition of traffic flow stability (based the on macroscopic traffic flow description) and related it to string stability (based on a car-following model). In Zhang (1999), traffic stability analyses are presented for a new second-order continuum traffic model introduced in that paper and for the PW model. Most of these previous works are based on deterministic traffic models. Recently, del Castillo (2001) discussed the propagation of perturbations in dense traffic flow using a stochastic approximation approach and a microscopic model.

The contributions of this paper are twofold. First, a wavefront expansion technique is applied to an entire class of second-order continuous traffic flow models, which allows us to analyze the shock wave propagation properties of these models. The relevance of this expansion technique lies in the fact that it is a nonlinear technique and, for that reason, allows us to precisely find what are the global stability conditions under which a perturbation travels along a highway. Using this technique, it is possible to verify previously established traffic flow stability results in the literature, which were obtained with linear and local approximation techniques. In most cases, the newly results in this paper are in agreement with what has been reported in the literature. In some cases, however, the conditions derived in this paper for traffic flow stability are more precise than those previously reported. Moreover, in this paper we also determine that, in most second-order models, downstream-moving waves decay quickly and do not significantly affect overall traffic flow. The existence of downstream-moving waves was cited by Daganzo (1995b) as a major deficiency of these models, since such waves do not occur in real traffic. The second contribution of the paper is on the use of a numerical tool that employs this wavefront expansion technique to simulate traffic flow. The numerical simulations results are more accurate and confirm the theoretical findings that explain why, with a proper choice of parameters, most second-order models can lead to appropriate results in terms of traffic flow stability prediction.

This paper consists of six parts. In Section 2, several different macroscopic traffic flow models are reviewed and a definition of stability of traffic flow is presented. Section 3 discusses the traffic flow characteristics. The stability results using a wavefront expansion technique are presented in Section 4. Traffic stability criteria are analyzed using a wavefront expansion technique, and comparisons among different models are discussed. Section 5 presents some numerical examples to illustrate the stability results in Section 4. Concluding remarks are presented in Section 6. 


\section{Macroscopic traffic flow models and stability}

In the past 50 years, a significant amount of research has been done on macroscopic modeling of traffic flows. In this paper we consider only a one lane highway without any on- or off-ramps. Let $\rho(x, t)$ denote the highway density, $q(x, t)$ the flow and $v(x, t)$ the traffic velocity at position $x$ along the highway at time $t$, respectively. By definition, $q(x, t)=\rho(x, t) v(x, t)$. Note that the traffic velocity $v(x, t)$ is the average speed of all vehicles on the highway around $x$. Conservation of vehicles on the highway gives us the following equation ${ }^{4}$

$$
\frac{\partial \rho}{\partial t}+\frac{\partial(\rho v)}{\partial x}=0
$$

and the velocity dynamics for various models can be written, in the following general form

$$
\frac{\partial v}{\partial t}+v \frac{\partial v}{\partial x}=-\frac{1}{\rho} \frac{\partial \mathscr{P}}{\partial x}+\frac{1}{\tau}\left(V_{\mathrm{e}}-v\right)
$$

where $V_{\mathrm{e}}$ is the generalized equilibrium velocity, which is given by the steady-state relationship between highway velocity $v$ and density $\rho$ (e.g. fundamental diagrams), $\mathscr{P}$ is the so-called traffic pressure, and $\tau$ is the so-called relaxation time, which is the time constant of the regulating traffic velocity $v$ to the equilibrium velocity $V_{\mathrm{e}}$.

For various macroscopic models, the traffic pressure $\mathscr{P}:=\mathscr{P}(\rho, v)$, relaxation time $\tau$, and the generalized equilibrium velocity $V_{\mathrm{e}}(\rho, v)^{5}$ are different:

1. If $\tau=0, \mathscr{P}=0$, we obtain the LWR first-order model (Lighthill and Whitham, 1955; Richard, 1956). ${ }^{6}$

2. If $\mathscr{P}=-V_{\mathrm{e}}(\rho) / 2 \tau$ with $V_{\mathrm{e}}(\rho, v)=V_{\mathrm{e}}(\rho)$, we obtain the PW model (Payne, 1971; Whitham, 1974).

3. Setting $\mathscr{P}=\rho \Theta$ with $\Theta=\Theta_{0}\left(1-\left(\rho / \rho_{\max }\right)\right)$ results in the model given in Phillips (1979).

4. If $\mathscr{P}=\rho \Theta_{0}-\eta(\partial v / \partial x)$, where $\eta$ is the so-called viscosity coefficient, we obtain the model given in Kerner and Konhäuser (1993).

5. If $\mathscr{P}=(v /(\gamma+2)) \rho^{\gamma+2}$, where $v$ is an anticipation parameter and $\gamma$ a dimensionless constant; and $V_{\mathrm{e}}(\rho)=V_{\mathrm{f}}$, where $V_{\mathrm{f}}$ is the free flow velocity; we obtain the model given in Michalopoulos et al. (1993). A similar model to that of Zhang (1998) is obtained for $\mathscr{P}=(1 / 3) \rho^{3} V_{\mathrm{e}}^{\prime 2}(\rho){ }^{7}$ with $V_{\mathrm{e}}^{\prime}(\rho)=\mathrm{d} V_{\mathrm{e}}(\rho) / \mathrm{d} \rho$.

\footnotetext{
${ }^{3}$ We can drop this assumption by adding relaxation terms in the conservation laws.

${ }^{4}$ In this paper we assume that the traffic flow variables $\rho(x, t), q(x, t)$ and $v(x, t)$ are differentiable.

${ }^{5}$ For most second-order traffic flow models, $V_{\mathrm{e}}$ is only a function of density $\rho$, i.e. $V_{\mathrm{e}}(\rho, v)=V_{\mathrm{e}}(\rho)$. However, here we extend this assumption, and consider $V_{\mathrm{e}}(v, t)$ to be a function of both density $\rho$ and flow velocity $v$, in order to accommodate various models.

${ }^{6}$ To see this fact, multiply $\tau$ on both sides of Eq. (2) and let $\tau=0$. Then we obtain $v=V_{\mathrm{e}}$. Combining Eq. (1) and $v=V_{\mathrm{e}}$ gives the LWR traffic model.

${ }^{7}$ In this paper, for simplicity, we consider $V_{\mathrm{e}}(\rho)$ to be piecewise linear in Zhang's model, thus $V_{\mathrm{e}}^{\prime \prime}(\rho)=0$. If other complicated functions are used for the fundamental diagram, the results are similar but are more difficult to compute.
} 
6. If $\mathscr{P}=\rho A v^{2}$, where $A=A(\rho)$ is a density-dependent function, and

$$
V_{\mathrm{e}}(\rho, v)=V_{\mathrm{e}}(\rho)\left[1-\frac{A}{A\left(\rho_{\max }\right)}\left(\frac{\rho_{a} T v}{1-\rho_{a} / \rho_{\max }}\right)^{2} B\left(\delta_{v}\right)\right],
$$

where $\rho_{a}$ is the density at point $x_{a}$ ahead of $x, B\left(\delta_{v}\right)$ is a macroscopic interaction term and $V_{\mathrm{e}}(\rho)$ is the normal fundamental diagram relationship between $v$ and $\rho$, we obtain the model given in Treiber et al. (1999).

We only listed a few continuous traffic flow models above. There exists a class of traffic flow models, which are given in the form of discrete space domain and continuous time domain equations, such as those presented by Cremer and Papageorgiou (1981), Papageorgiou et al. (1989) and Daganzo (1994). These models are similar to the above continuous models, but use space discretization and incorporate other modifications, such as nonlinear density saturation functions, etc.

Each of these models has been tested either by simulation or against actual traffic flow measurements. In this paper we will not discuss or evaluate which traffic model more accurately fits real traffic data. Instead, we will analyzed the stability properties of all these second-order models under perturbed initial conditions, assuming dense traffic flow. Since second-order traffic models have been and continue to be widely used for traffic flow studies for various purposes, we believe that a systematic investigation of their stability properties, under several equilibrium states, is important to better understand their intrinsic properties. A few stability results using linearization techniques have been reported in the literature for some specific second-order traffic models, such as the Whitham (1974) and Zhang (1999) models. However, prior to this paper, there did not exist a unified nonlinear study of second-order traffic models. This paper fills this gap.

First, we should define the stability of the system described by Eqs. (1) and (2). In this paper, we mainly focus on the following definition of traffic flow propagation stability.

Definition 1 (Traffic flow propagation stability). Let $\mathbf{q}_{\mathrm{e}}(x, t)=\left[\rho_{\mathrm{e}}(x, t), v_{\mathrm{e}}(x, t)\right]^{\mathrm{T}}$ denote the nominal equilibrium state of the traffic system on a highway with length $L$. Let $\mathbf{q}_{\mathrm{p}}(x, t)$ be a perturbed state. The traffic flow $\mathbf{q}_{\mathrm{e}}(x, t)$ is propagation stable under perturbation traffic state $\mathbf{q}_{\mathrm{p}}$ if the spatial gradient of the perturbed state is bounded, i.e. $\left\|\left(\partial q_{\mathrm{p}} / \partial x\right)(x, t)\right\|<\infty$, for $\forall t>0, x \in[0, L]$. If, in addition to the above, $\lim _{t \rightarrow \infty}\left\|\left(\partial q_{\mathrm{p}} / \partial x\right)(x, t)\right\|=0$, then, the traffic state $\mathbf{q}_{\mathrm{e}}$ is asymptotically propagation stable.

The wavefront of a traffic system can be illustrated by Fig. 1. Consider a pair of equilibrium states, $\mathbf{q}_{\mathrm{e}}=\left[\rho_{\mathrm{e}}, v_{\mathrm{e}}\right]$, of the traffic system. If there is a perturbation at position $x_{0}$, then the wavefront is the propagation curve of such perturbation along the unperturbed density and velocity. If a traffic system is propagation stable, then the magnitude of the initial perturbation should not increase during its propagation. On the other hand, if a traffic system is propagation unstable, a perturbed density or velocity wave may increase in magnitude as it propagates and finally form a shock wave or bottleneck on the highway. In this paper, we focus on how a perturbed density or velocity profile propagates if we use a second-order model to model traffic behavior. 


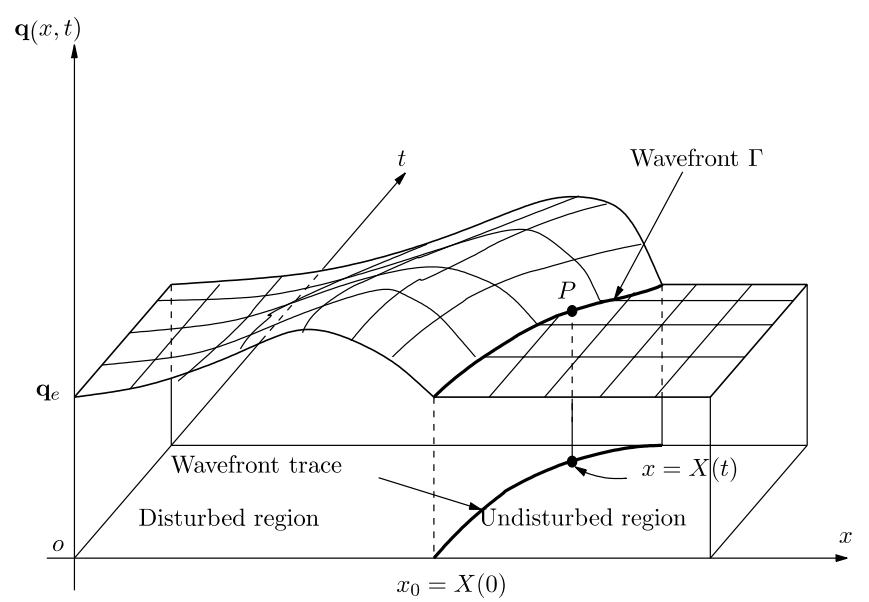

Fig. 1. A schematic of a solution surface, wavefront and wavefront trace with an initial $C^{1}$ discontinuous condition $\mathbf{q}\left(x_{0}, 0\right)$.

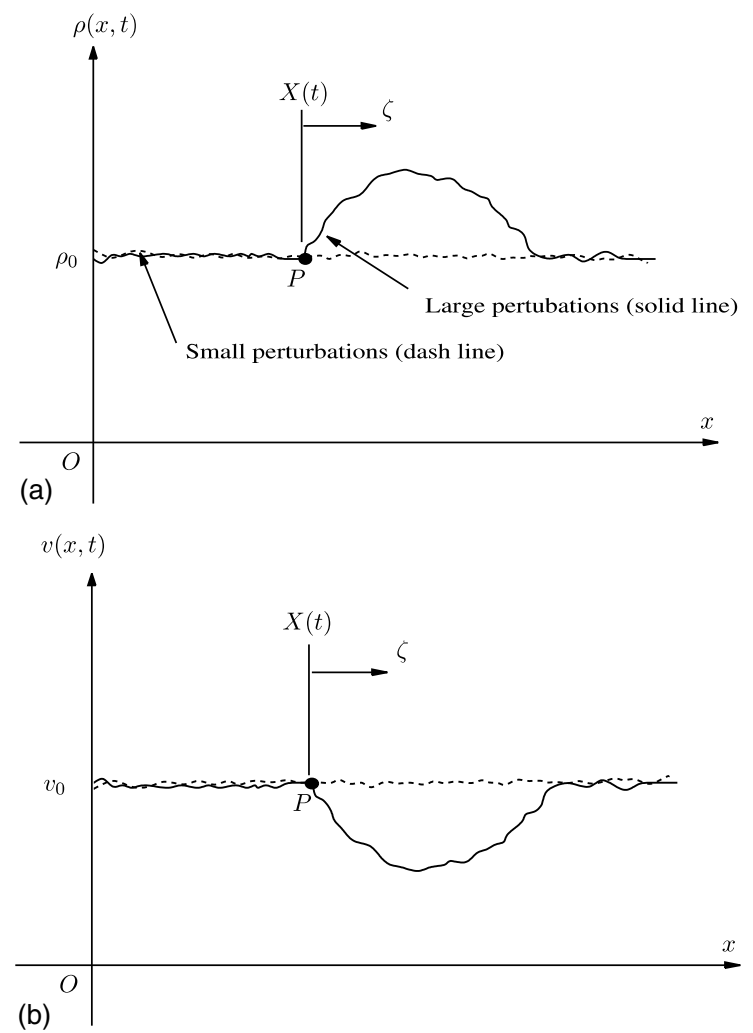

Fig. 2. A schematic of perturbed traffic density and velocity profiles (a) density $\rho(x, t)$; (b) velocity $v(x, t)$.

The above definition of traffic flow propagation stability is different from that used by Swaroop and Rajagopal (1999), who used the definition of stability in the sense of Lyapunov for distributed 
parameter dynamic systems. We believe that the traffic flow propagation stability definition used in this paper is more akin to that used by traffic engineers, who in general refer to traffic instability as the formation of a shock wave.

It is important to distinguish between small and large perturbations in the stability analysis of traffic systems (see Fig. 2). Most transportation researchers have used linearized methods to analyze traffic stability by assuming that the perturbations are small. For example, Whitham (1974), Swaroop and Rajagopal (1999) and Zhang (1999). The approximate linearization stability analysis of the nonlinear PDE system (1) and (2) is only valid when the magnitude of the perturbations is small, since the analysis neglects higher order terms. However, when the perturbation is fairly large, the linearization method may produce incorrect results as pointed out by Whitham (1974). Therefore, it is necessary to use other techniques to analyze traffic stability under large perturbations. In this paper, we discuss such an approach by utilizing a wavefront expansion technique.

\section{Traffic flow characteristics}

To discuss traffic flow stability we need to first investigate the characteristic velocity of a traffic system given by Eqs. (1) and (2). The importance of the so-called characteristic curves is that all perturbations propagate along these curves on the highway. Therefore, the characteristics of a traffic system give intrinsic information regarding wave propagation. This important fact and several methods for calculating the characteristics for specific second-order models have been reported in literature, e.g. Whitham (1974) and Zhang (1999). However, there is no systematic description of such a consideration in transportation engineering.

From now on, we use shortened notations to denote partial derivatives, for example, $\rho_{x}:=\partial \rho / \partial x$. We also assume that the traffic pressure function $\mathscr{P}(\rho, v): \mathbb{R}_{+} \times \mathbb{R}_{+} \mapsto \mathbb{R}$ is smooth, i.e. $\mathscr{P}:=\mathscr{P}(\rho, v) \in \mathbb{C}^{\infty}\left(\mathbb{R}_{+} \times \mathbb{R}_{+}\right)$. We thus rewrite Eq. (1) as

$$
\rho_{t}+v \rho_{x}+\rho v_{x}=0
$$

and using the fact $\mathscr{P}=\mathscr{P}(\rho, v)$, rewrite Eq. (2) as

$$
v_{t}+\frac{1}{\rho} \mathscr{P}_{\rho} \rho_{x}+\left(v+\frac{1}{\rho} \mathscr{P}_{v}\right) v_{x}=\frac{1}{\tau}\left(V_{\mathrm{e}}-v\right) .
$$

Consider the full differentials of $\rho(x, t)$ and $v(x, t)$ as follows:

$$
\begin{aligned}
& \mathrm{d} \rho(x, t)=\rho_{x} \mathrm{~d} x+\rho_{t} \mathrm{~d} t, \\
& \mathrm{~d} v(x, t)=v_{x} \mathrm{~d} x+v_{t} \mathrm{~d} t,
\end{aligned}
$$

and write Eqs. (3)-(6) in matrix form

$$
\left[\begin{array}{cccc}
1 & v & 0 & \rho \\
0 & \frac{1}{\rho} \mathscr{P}_{\rho} & 1 & \left(v+\frac{1}{\rho} \mathscr{P}_{v}\right) \\
\mathrm{d} t & \mathrm{~d} x & 0 & 0 \\
0 & 0 & \mathrm{~d} t & \mathrm{~d} x
\end{array}\right]\left[\begin{array}{c}
\rho_{t} \\
\rho_{x} \\
v_{t} \\
v_{x}
\end{array}\right]=\left[\begin{array}{c}
0 \\
\frac{1}{\tau}\left(V_{\mathrm{e}}-v\right) \\
\mathrm{d} \rho \\
\mathrm{d} v
\end{array}\right]
$$


To admit discontinuous solutions (shocks) for $\rho(x, t)$ and $v(x, t)$, which are observed in real traffic flow, it is necessary that the coefficient matrix of partial derivatives be singular.

Therefore, the characteristic velocities $v_{\mathrm{c}}$ are given by

$$
v_{\mathrm{c}}:=\frac{\mathrm{d} x}{\mathrm{~d} t}=v+\frac{1}{2 \rho} \mathscr{P}_{v} \pm \sqrt{\frac{1}{4 \rho^{2}}\left(\mathscr{P}_{v}\right)^{2}+\mathscr{P}_{\rho}} .
$$

For each of the different models discussed above, the characteristic velocities have the following formulae:

1. For the PW model, $\mathscr{P}=-V_{\mathrm{e}}(\rho) / 2 \tau$, and

$$
v_{\mathrm{c}}=v \pm \sqrt{-\frac{1}{2 \tau} \frac{\partial V_{\mathrm{e}}}{\partial \rho}}
$$

2. For Phillips's model, $\mathscr{P}=\Theta_{0} \rho\left(1-\left(\rho / \rho_{\max }\right)\right)$ and

$$
v_{\mathrm{c}}=v \pm \sqrt{\Theta_{0}\left(1-\frac{2 \rho}{\rho_{\max }}\right)} .
$$

3. For Michalopoulos' model $\mathscr{P}=(v / \gamma+2) \rho^{\gamma+2}$ and

$$
v_{\mathrm{c}}=v \pm \sqrt{v \rho^{\gamma+1}}
$$

and, for Zhang's model, $\mathscr{P}=(1 / 3) \rho^{3} V_{\mathrm{e}}^{\prime} 2(\rho)$ and

$$
v_{\mathrm{c}}=v \pm\left|V_{\mathrm{e}}^{\prime}(\rho)\right| \rho \text {. }
$$

4. For Treiber's model, $\mathscr{P}=\rho A(\rho) v^{2}$ and

$$
v_{\mathrm{c}}=v\left[1+A(\rho) \pm \sqrt{A^{2}(\rho)+A+\rho A^{\prime}(\rho)}\right] \text {. }
$$

Remark 1. From the above calculations we find that, for all the second-order traffic flow models considered in this paper, one of the solutions of the characteristic velocity is larger than the average traffic flow velocity $v$. This is not realistic, since shock waves do not propagate in the downstream direction in actual highways. This fact has been criticized by Daganzo (1995b) as a major deficiency of most second-order models. Several researchers, such as Liu et al. (1998) and del Castillo et al. (1994), have proposed some modified second-order models in which all characteristic velocities are smaller than the average vehicle traveling velocity. However, such modifications are limited to some specific situations and the resulting modified models lack physical interpretations. From Eq. (7), the characteristic velocity $v_{\mathrm{c}} \leqslant v$ iff

$$
\mathscr{P}_{v} \leqslant 0, \quad \mathscr{P}_{\rho} \leqslant 0 \quad \text { and } \quad \frac{1}{4 \rho^{2}} \mathscr{P}_{v}^{2}+\mathscr{P}_{\rho} \geqslant 0 .
$$

Even though there is such a major deficiency as discussed above, we investigate the stability conditions for the various second-order traffic models in this paper because most second-order models have been applied to either simulation tests or real traffic measurements and have been validated to capture some important properties of traffic systems. More importantly, in this paper we find that, for most second-order traffic models, the perturbation that travels faster than traffic 
decays quickly. Numerical examples in Section 5 illustrate this fact. Therefore, we believe that the effect of such a deficiency in some second-order models may not be significant under proper tuning of the model parameters.

\section{Stability results}

In this section we discuss the propagation stability conditions for second-order traffic flow models (Eqs. (1) and (2)) under large perturbations. Here we consider a fairly large perturbation around the equilibrium density $\rho_{\mathrm{e}}$ and average velocity $V_{\mathrm{e}}$. Fig. 2 shows a schematic of such a perturbation versus small perturbations. Suppose that traffic has a constant traffic density $\rho_{0}=\rho_{\mathrm{e}}$ and velocity $v_{0}=V_{\mathrm{e}}\left(\rho_{\mathrm{e}}\right)$, where the $\left(\rho_{\mathrm{e}}, V_{\mathrm{e}}\left(\rho_{\mathrm{e}}\right)\right)$ pair is on the fundamental diagram. Obviously, $\rho_{0}, v_{0}$ are the solutions of Eqs. (1) and (2). Assume a disturbance, such as a "bump" in Fig. 2, around the density $\rho_{0}$ and its corresponding equilibrium velocity $v_{0}$. Without loss of generality, assume that the first derivative of the density profile around the wavefront $P$ is discontinuous. ${ }^{8}$

It is convenient to expand the solution of the system around the wavefront in powers of

$$
\zeta=x-X(t),
$$

where $X(t)$ is the location of the wavefront $P$ at time $t$ and the wavefront has the characteristic velocity $v_{\mathrm{c}}$ at the equilibrium states, i.e.

$$
\dot{X}(t)=v_{\mathrm{c}}\left(\rho_{0}, v_{0}\right)=v_{0}+u_{0}=v_{0}+\frac{1}{2 \rho_{0}} \mathscr{P}_{v}^{0} \pm \sqrt{\frac{1}{4 \rho_{0}^{2}}\left(\mathscr{P}_{v}^{0}\right)^{2}+\mathscr{P}_{\rho}^{0}}
$$

and

$$
u_{0}:=\frac{1}{2 \rho_{0}} \mathscr{P}_{v}^{0} \pm \sqrt{\frac{1}{4 \rho_{0}^{2}}\left(\mathscr{P}_{v}^{0}\right)^{2}+\mathscr{P}_{\rho}^{0}}, \quad \mathscr{P}_{v}^{0}=\left.\mathscr{P}_{v}\right|_{\left(\rho_{0}, v_{0}\right)} \quad \text { and } \quad \mathscr{P}_{\rho}^{0}=\left.\mathscr{P}_{\rho}\right|_{\left(\rho_{0}, v_{0}\right)}
$$

Using Eq. (9), we can expand the flow variables $\rho$ and $v$ behind the wavefront in a power series of $\zeta$ as

$$
\begin{aligned}
& \rho(x, t)=\rho_{0}+\zeta \rho_{1}(t)+\frac{1}{2} \zeta^{2} \rho_{2}(t)+\cdots, \\
& v(x, t)=v_{0}+\zeta v_{1}(t)+\frac{1}{2} \zeta^{2} v_{2}(t)+\cdots,
\end{aligned}
$$

where

$$
\rho_{i}(t)=\left.\frac{\partial^{i} \rho}{\partial x^{i}}\right|_{\left(X(t)^{-}, t\right)}, \quad v_{i}(t)=\left.\frac{\partial^{i} v}{\partial x^{i}}\right|_{\left(X(t)^{-}, t\right)}, \quad i=1,2,3, \ldots
$$

We now calculate the partial derivatives of state variables $\rho$ and $v$, using Eqs. (11) and (12),

$$
\rho_{t}=-\dot{X}(t) \rho_{1}(t)+\zeta \dot{\rho}_{1}(t)+\zeta[-\dot{X}(t)] \rho_{2}(t)+\frac{1}{2} \zeta^{2} \dot{\rho}_{2}(t)+\cdots,
$$

\footnotetext{
${ }^{8}$ This assumption can be generalized. For example, if the $m$ th derivatives of $\rho$ and $v$ are the first ones to be discontinuous, the expanded power series (Eqs. (11) and (12)) beyond $\rho_{0}$ and $v_{0}$ start with the term in $\zeta^{m}$.
} 


$$
\begin{aligned}
& \rho_{x}=\rho_{1}(t)+\zeta \rho_{2}(t)+\frac{1}{2} \zeta^{2} \rho_{3}(t)+\cdots, \\
& v_{t}=-\dot{X}(t) v_{1}(t)+\zeta \dot{v}_{1}(t)+\zeta[-\dot{X}(t)] v_{2}(t)+\frac{1}{2} \zeta^{2} \dot{v}_{2}(t)+\cdots, \\
& v_{x}=v_{1}(t)+\zeta v_{2}(t)+\frac{1}{2} \zeta^{2} v_{3}(t)+\cdots
\end{aligned}
$$

Similarly, for $\mathscr{P}$ and $V_{\mathrm{e}}(\rho)$, we obtain

$$
\begin{aligned}
& \mathscr{P}_{v}=\mathscr{P}_{v}^{0}+\zeta\left[\mathscr{P}_{v \rho}^{0} \rho_{1}(t)+\mathscr{P}_{v v}^{0} v_{1}(t)\right]+\cdots, \\
& \mathscr{P}_{\rho}=\mathscr{P}_{\rho}^{0}+\zeta\left[\mathscr{P}_{\rho \rho}^{0} \rho_{1}(t)+\mathscr{P}_{\rho v}^{0} v_{1}(t)\right]+\cdots, \\
& V_{\mathrm{e}}(\rho, v)=V_{\mathrm{e}}^{0}+\zeta\left[\left(V_{\mathrm{e}}\right)_{\rho}^{0} \rho_{1}(t)+\left(V_{\mathrm{e}}\right)_{v}^{0} v_{1}(t)\right]+\cdots,
\end{aligned}
$$

where

$$
V_{\mathrm{e}}^{0}:=V_{\mathrm{e}}\left(\rho_{0}\right), \quad\left(V_{\mathrm{e}}\right)_{\rho}^{0}:=\left.\frac{\partial V_{\mathrm{e}}}{\partial \rho}\right|_{\left(\rho_{0}, v_{0}\right)} \quad \text { and } \quad\left(V_{\mathrm{e}}\right)_{v}^{0}:=\left.\frac{\partial V_{\mathrm{e}}}{\partial v}\right|_{\left(\rho_{0}, v_{0}\right)} .
$$

Substituting Eqs. (13a)-(13d) and (10) into Eq. (3) and Eqs. (13a)-(13c) and (14a)-(14c) into Eq. (4), for the coefficients of the first two terms $\zeta^{0}$ and $\zeta^{1}$, we obtain

$$
\begin{aligned}
& -\rho_{1} u_{0}+\rho_{0} v_{1}=0, \\
& \dot{\rho}_{1}-\rho_{2} u_{0}+2 \rho_{1} v_{1}+\rho_{0} v_{2}=0, \\
& -\rho_{0} v_{1} u_{0}+v_{1} \mathscr{P}_{v}^{0}+\rho_{1} \mathscr{P}_{\rho}^{0}=0, \\
& \rho_{0} \dot{\rho}_{1}+v_{2}\left(\mathscr{P}_{v}^{0}-\rho_{0} u_{0}\right)+\rho_{2} \mathscr{P}_{\rho}^{0}+\rho_{1} v_{1}\left(2 \mathscr{P}_{\rho v}^{0}-u_{0}\right)+v_{1}^{2}\left(\rho_{0}+\mathscr{P}_{v v}^{0}\right)+\rho_{1}^{2} \mathscr{P}_{\rho \rho}^{0} \\
& \quad+\frac{1}{\tau}\left[v_{1}-\left(V_{\mathrm{e}}\right)_{\rho}^{0} \rho_{1}-\left(V_{\mathrm{e}}\right)_{v}^{0} v_{1}\right]=0 .
\end{aligned}
$$

For Eqs. (15b) and (15d), notice that the coefficients of terms $\rho_{2}$ and $v_{2}$ are linearly dependent since

$$
\operatorname{det}\left[\begin{array}{cc}
-u_{0} & \rho_{0} \\
\mathscr{P}_{\rho}^{0} & \mathscr{P}_{v}^{0}-\rho_{0} u_{0}
\end{array}\right]=\rho_{0} u_{0}^{2}-\mathscr{P}_{v}^{0} u_{0}-\rho_{0} \mathscr{P}_{\rho}^{0}=0
$$

and therefore $\rho_{2}$ and $v_{2}$ can be eliminated. Plugging $\rho_{1}=\left(\rho_{0} / u_{0}\right) v_{1}$ in Eqs. (15b) and (15d), we obtain

$$
\dot{v}_{1}+\alpha v_{1}+\beta v_{1}^{2}=0
$$

where

$$
\alpha=\frac{\rho_{0} u_{0}}{\tau\left(2 \rho_{0} u_{0}-\mathscr{P}_{v}^{0}\right)}\left(1-\left(V_{\mathrm{e}}\right)_{v}^{0}-\frac{\left(V_{\mathrm{e}}\right)_{\rho}^{0} \rho_{0}}{u_{0}}\right), \quad \beta=\frac{\left(\rho_{0} \frac{\partial}{\partial \rho}+u_{0} \frac{\partial}{\partial v}\right)^{2} \mathscr{P}^{0}+2 \rho_{0} \mathscr{P}_{\rho}^{0}}{u_{0}\left(2 \rho_{0} u_{0}-\mathscr{P}_{v}^{0}\right)}
$$

Notice that

$$
v_{1}(t)=\left.\frac{\partial v(x, t)}{\partial x}\right|_{\left(X(t)^{-}, t\right)^{-}},
$$


Table 1

Stability conditions for the system given by Eq. (16)

\begin{tabular}{lll}
\hline Parameters $\alpha$ and $\beta$ & Stable region & Unstable region \\
\hline$\beta>0, \alpha>0$ & $v_{1}(0) \in[-\alpha / \beta, \infty), v_{1}(t) \rightarrow 0$ & $v_{1}(0) \in(-\infty,-\alpha / \beta), v_{1}(t) \rightarrow-\infty$ \\
$\beta>0, \alpha=0$ & $v_{1}(0) \in \mathbb{R}, v_{1}(t) \rightarrow 0$ & $\emptyset$ \\
$\beta>0, \alpha<0$ & $v_{1}(0) \in[0, \infty), v_{1}(t) \rightarrow-\alpha / \beta$ & $v_{1}(0) \in(-\infty, 0), v_{1}(t) \rightarrow-\infty$ \\
$\beta<0, \alpha=0$ & $\emptyset$ & $v_{1}(0) \in \mathbb{R}, v_{1}(t) \rightarrow \infty$ \\
$\beta<0, \alpha>0$ & $v_{1}(0) \in(-\infty,-\alpha / \beta), v_{1}(t) \rightarrow 0$ & $v_{1}(0) \in(-\alpha / \beta, \infty), v_{1}(t) \rightarrow \infty$ \\
$\beta<0, \alpha<0$ & $v_{1}(0) \in(-\infty, 0), v_{1}(t) \rightarrow-\alpha / \beta$ & $v_{1}(0) \in(0, \infty), v_{1}(t) \rightarrow \infty$ \\
$\beta=0, \alpha>0$ & $v_{1}(0) \in \mathbb{R}, v_{1}(t) \rightarrow 0$ & $\emptyset$ \\
$\beta=0, \alpha<0$ & $\emptyset$ & $v_{1}(0) \in \mathbb{R}, v_{1}(t) \rightarrow \infty$ \\
\hline
\end{tabular}

is namely the slope of the wavefront at point $P$ (in Fig. 2). The above Riccati equation gives the slope evolution at the wavefront. The propagation stability of Eq. (16) can thus be analyzed in terms of the initial condition $v_{1}(0)$ and the parameters $\alpha$ and $\beta$. Table 1 shows the stability conditions of the system given by Eq. (16). Moreover, the solution of the Riccati equation (16) is given as

$$
v_{1}(t)=\frac{\alpha}{\beta} \frac{\mathrm{e}^{-\alpha t}}{\left(1+\frac{\alpha}{\beta v_{1}(0)}\right)-\mathrm{e}^{-\alpha t}},
$$

where $v_{1}(0)$ is the initial condition for $v_{1}(t)$ at $t=0$.

For the second-order traffic flow models that were discussed in Section 2, we can use the above results to calculate the stability criterion for various models. There are two different characteristic velocities for second-order traffic models: one moving downstream at speed $v_{0}+u_{01}\left(>v_{0}\right)$ and another moving upstream at speed $v_{0}+u_{02}\left(<v_{0}\right)$, where $u_{01}$ and $u_{02}$ are two values of $u_{0}$ given by Eq. (10).

Notice that, for the downstream-moving characteristic velocity $v_{\mathrm{c}}=v_{0}+u_{01}$ with $u_{01}>0$, and for most second-order traffic models, $\mathscr{P}_{v}^{0}=0,\left(V_{\mathrm{e}}\right)_{v}^{0}=0$ and $\left(V_{\mathrm{e}}\right)_{\rho}^{0}<0$. Thus,

$$
\alpha=\frac{1}{2 \tau}\left(1+\frac{\left|\left(V_{\mathrm{e}}\right)_{\rho}^{0}\right| \rho_{0}}{u_{0}}\right) \gg 1, \quad \beta>0 .
$$

As a consequence the magnitude of a downstream-moving perturbation at characteristic speed $v_{0}+u_{01}$ decays to zero quickly, since $v_{1}(t) \rightarrow 0$ quickly by the solution (17) for $v_{1}(0) \geqslant-\alpha / \beta$. We can therefore neglect the effect of the forward moving branch of the perturbation for most secondorder traffic models.

In the following, we consider the solution branch for which the characteristic velocity $v_{0}+u_{02}$ satisfies $u_{02}<0$, i.e. the upstream moving branch.

1. For the PW model,

$$
\mathscr{P}=-\frac{V_{\mathrm{e}}(\rho)}{2 \tau}, \quad u_{02}=-\sqrt{-\frac{V_{\mathrm{e}}^{\prime}\left(\rho_{0}\right)}{2 \tau}}
$$

and

$$
\alpha=\frac{1}{2 \tau}\left(1-\rho_{0} \sqrt{-2 V_{\mathrm{e}}^{\prime}\left(\rho_{0}\right) \tau}\right), \quad \beta=1 .
$$


Thus, from Table 1 in order for traffic flow perturbations to have stability for any initial condition $v_{1}(0)>-\alpha / \beta$, we need $\alpha \geqslant 0$. Therefore we obtain the condition

$$
1-\rho_{0} \sqrt{-2 V_{\mathrm{e}}^{\prime}\left(\rho_{0}\right) \tau} \geqslant 0
$$

which can be rearranged as

$$
\frac{1}{2} \geqslant-\rho_{0}^{2} V_{\mathrm{e}}^{\prime}\left(\rho_{0}\right) \tau \text {. }
$$

The "negative" sign in the above inequality arises because $V_{\mathrm{e}}^{\prime}(\rho) \leqslant 0$. Condition (18) is the same as that given in Whitham (1974) and Holland (1998). However, in Whitham (1974) and Holland (1998), the stability conditions were derived using an approximate linearized stability analysis, and a microscopic traffic model, i.e. a car-following model, respectively.

2. For Philips's model,

$$
\mathscr{P}=\rho \Theta_{0}\left(1-\frac{\rho}{\rho_{\max }}\right), \quad u_{02}=-\sqrt{\Theta_{0}\left(1-\frac{2 \rho}{\rho_{\max }}\right)},
$$

and

$$
\alpha=\frac{1}{2 \tau}\left(1+\frac{\rho_{0} V_{\mathrm{e}}^{\prime}\left(\rho_{0}\right)}{\sqrt{\Theta_{0}\left(1-\frac{2 \rho_{0}}{\rho_{\max }}\right)}}\right), \quad \beta=\frac{\rho_{\max }-3 \rho_{0}}{\rho_{\max }-2 \rho_{0}} .
$$

Assume that $\rho_{0} \leqslant \rho_{\max } / 3$, then $\beta \geqslant 0$. In order to have stability for any initial condition $v_{1}(0)>$ $-\alpha / \beta$, we need $\alpha \geqslant 0$, therefore the stability condition is

$$
\rho_{0} \leqslant \sqrt{\Theta_{0}+\left(\frac{\Theta_{0}}{\rho_{\max } V_{\mathrm{e}}^{\prime^{2}}\left(\rho_{0}\right)}\right)^{2}}-\frac{\Theta_{0}}{\rho_{\max } V_{\mathrm{e}}^{\prime 2}\left(\rho_{0}\right)} .
$$

3. For Michalopoulos' model, $\mathscr{P}=(v /(\gamma+2)) \rho^{\gamma+2}, u_{02}=-\sqrt{v \rho^{\gamma+1}}$. Noticing that $V_{\mathrm{e}}=V_{\mathrm{f}}$ is independent of $\rho$ and $v$, we have

$$
\alpha=\frac{1}{2 \tau}, \quad \beta=\frac{\gamma+3}{2} .
$$

Thus, for any initial conditions $v_{1}(0)>-\alpha / \beta$, the system is stable.

For Zhang's model, $\mathscr{P}=(1 / 3) \rho^{3} V_{\mathrm{e}}^{\prime} 2, u_{02}=-\left|V_{\mathrm{e}}^{\prime}(\rho)\right| \rho$ and

$$
\alpha=\frac{1}{\tau}, \quad \beta=2 .
$$

Since $\beta>0, \alpha>0$, the slope of the perturbations around the wavefront converges to zero for $v_{1}(0)>-\alpha / \beta$, according to Table 1 . This result is similar to the stability criterion found in Zhang (1999). In Zhang (1999) the author claimed that the new model was "inherently stable, as is the LWR model, in the sense that the magnitude of a small disturbance in the traffic stream does not grow without a bound". A linearized approximation was used to find the stability result in Zhang (1999). However, the stability results that were derived in this section for Zhang's model 
are more precise since the slope magnitude of the disturbance does asymptotically converge to zero.

4. For the gas-kinetic-based traffic model (GKT model) proposed in Treiber et al. (1999), $\mathscr{P}=\rho A(\rho) v^{2}$ and $u_{02}=v_{0}\left[A\left(\rho_{0}\right)-\sqrt{A^{2}\left(\rho_{0}\right)+A\left(\rho_{0}\right)+\rho_{0} A^{\prime}\left(\rho_{0}\right)}\right]$. In Treiber et al. (1999), the density-dependent function $A(\rho)$ and generalized equilibrium velocity $V_{\mathrm{e}}(\rho, v)$ are given by

$$
\begin{aligned}
& A(\rho)=A_{0}+\Delta A\left[\tanh \left(\frac{\rho-\rho_{\mathrm{c}}}{\Delta \rho}\right)+1\right], \\
& V_{\mathrm{e}}(\rho, v)=V_{\mathrm{e}}(\rho)\left[1-\frac{A(\rho)}{2 A\left(\rho_{\max }\right)}\left(\frac{\rho_{a} T v}{1-\rho_{a} / \rho_{\max }}\right)^{2} B\left(\delta_{v}\right)\right] .
\end{aligned}
$$

Thus,

$$
\begin{aligned}
& \alpha=\frac{\sqrt{A_{0}^{2}+A_{0}+\rho_{0} A_{0}^{\prime}}-A_{0}}{\tau \sqrt{A_{0}^{2}+A_{0}+\rho_{0} A_{0}^{\prime}}}\left\{1-\frac{\rho_{0} V_{\mathrm{e}}^{\prime}\left(\rho_{0}\right)}{V_{\mathrm{e}}(\rho) u_{0}}+\frac{B\left(\delta_{v}\right)}{\rho_{\max }}\left(\frac{\rho_{a} T V_{\mathrm{e}}\left(\rho_{0}\right)}{1-\rho_{a} / \rho_{\max }}\right)^{2}\left[A_{0}+\frac{\rho_{0} A_{0}^{\prime}}{2 u_{0}}+\frac{A_{0} \rho_{0} V_{\mathrm{e}}^{\prime}\left(\rho_{0}\right)}{V_{\mathrm{e}}\left(\rho_{0}\right) u_{0}}\right]\right\}, \\
& \beta=\frac{1}{\tau \sqrt{A_{0}^{2}+A_{0}+\rho_{0} A_{0}^{\prime}}\left(\sqrt{A_{0}^{2}+A_{0}+\rho_{0} A_{0}^{\prime}}-A_{0}\right)}\left[\left(\rho_{0} \frac{\partial}{\partial \rho}+u_{0} \frac{\partial}{\partial v}\right)^{2} \mathscr{P}^{0}+2 \rho_{0} \mathscr{P}_{\rho}^{0}\right]>0 .
\end{aligned}
$$

where $A_{0}:=A\left(\rho_{0}\right)$ and $A_{0}^{\prime}:=A^{\prime}\left(\rho_{0}\right)$. Considering $\beta>0$ and the stability condition in Table 1 for $v_{1}(0)>-\alpha / \beta$, we need

$$
1-\frac{\rho_{0} V_{\mathrm{e}}^{\prime}\left(\rho_{0}\right)}{V_{\mathrm{e}}\left(\rho_{0}\right) u_{0}}+\frac{B\left(\delta_{v}\right)}{\rho_{\max }}\left(\frac{\rho_{a} T V_{\mathrm{e}}\left(\rho_{0}\right)}{1-\rho_{a} / \rho_{\max }}\right)^{2}\left[A_{0}+\frac{\rho_{0} A_{0}^{\prime}}{2 u_{0}}+\frac{A_{0} \rho_{0} V_{\mathrm{e}}^{\prime}\left(\rho_{0}\right)}{V_{\mathrm{e}}\left(\rho_{0}\right) u_{0}}\right]>0 .
$$

If $\rho_{0}$ satisfies the above condition, we can guarantee traffic flow propagation stability.

For most second-order traffic models, $\alpha>0, \beta>0$. If the initial slope of the velocity perturbation $v_{1}(0)$ satisfies $v_{1}(0) \geqslant-\alpha / \beta$, then the magnitude of the perturbation reduces to zero and perturbed traffic returns to the equilibrium state. If $v_{1}(0)<-\alpha / \beta$, then the perturbation will form a shock wave. We can estimate the time $t_{\mathrm{f}}$ from $t=0$ when a shock wave has been formed as

$$
t_{\mathrm{f}}=-\frac{1}{\alpha} \ln \left(1+\frac{\alpha}{\beta} \frac{1}{v_{1}(0)}\right)
$$

since $v_{1}\left(t_{\mathrm{f}}\right) \rightarrow-\infty$ using Eq. (17).

In the above analysis, we did not discuss the stability property of the first-order LWR model because it has been shown that the LWR model propagates any perturbation upstream with characteristic velocity $v_{\mathrm{c}}=V_{\mathrm{e}}(\rho)+\rho V_{\mathrm{e}}^{\prime}(\rho)\left(<V_{\mathrm{e}}(\rho)\right)$ (Whitham, 1974; LeVeque, 1992). This result can be easily obtained using the techniques presented in this section. We have however not applied the wavefront expansion technique to the kinetic model proposed by Kerner and Konhäuser (1993), due to the fact that the existence of the viscosity term $\partial^{2} v / \partial x^{2}$ in that model will smooth the wavefront even though the so-called viscosity coefficient $\eta$ may be tiny. Therefore, the proposed technique will not give us a correct prediction. A linearized stability condition has been given by Kerner and Konhäuser (1993) for a circular highway. 


\section{Numerical examples}

In this section we illustrate the results of the previous section with some numerical examples. Computing numerical solutions for conservation equations such as Eqs. (1) and (2) is difficult because of the subtlety involved in capturing discontinuous solutions such as shock waves. There are different numerical schemes for traffic flow computations, such as the finite difference methods used in Daganzo (1995a), Helbing and Treiber (1999), Liu et al. (1997), and Roe's flux difference splitting method by Leo and Pretty (1992). More details on numerical methods for conservation laws can be found in LeVeque (1992).

CLAWPACK LeVeque (2000) is one of these packages which have been developed recently using more accurate numerical methods for conservation laws. It is based on the wave propagation algorithms of LeVeque (1997). Rewriting the macroscopic traffic model (1) and (2) in a conservation form as follows:

$$
\left[\begin{array}{c}
\rho \\
\rho v
\end{array}\right]_{t}+\left[\begin{array}{c}
\rho v \\
\rho v^{2}+\mathscr{P}
\end{array}\right]_{x}=\left[\begin{array}{c}
0 \\
\frac{\rho}{\tau}\left(V_{\mathrm{e}}-v\right)
\end{array}\right]
$$

and denoting $\mathbf{p}=[\rho \rho v]^{\mathrm{T}} \in \mathbb{R}^{2}, \mathbf{f}(\mathbf{p})=\left[\rho v \rho v^{2}+\mathscr{P}\right]^{\mathrm{T}} \in \mathbb{R}^{2}$ and $\psi(\mathbf{p})=\left[0(\rho / \tau)\left(V_{\mathrm{e}}-v\right)\right]^{\mathrm{T}} \in \mathbb{R}^{2}$, Eq. (23) can be rewritten as

$$
\mathbf{p}_{t}+\mathbf{f}(\mathbf{p})_{x}=\psi(\mathbf{p})
$$

which is in the form that can be integrated by the CLAWPACK wave propagation algorithms.

In what follows, we apply this numerical scheme to the PW macroscopic traffic flow model. For the PW model, the parameters and fundamental diagram are taken from Table 2 (Payne, 1979).

In order to test the traffic flow stability condition that was obtained in the previous section, we need to first check the stability criterion (18). Since $\mu=\sqrt{-V_{\mathrm{e}}^{\prime}(\rho) / 2 \tau}=$ constant, we need to modify the stability criterion (18) as follows:

$$
1+\frac{V_{\mathrm{e}}^{\prime}\left(\rho_{0}\right) \rho_{0}}{\mu} \geqslant 0
$$

From Table 2 we can compute the critical density $\rho_{\mathrm{c}}$ by solving the above algebraic equation, with

$$
0 \leqslant \rho_{0} \leqslant 26 \mathrm{veh} / \mathrm{km} / \text { lane or } \rho_{\mathrm{cl}}=52.4 \leqslant \rho_{0} \leqslant \rho_{\mathrm{c} 2}=114.7 \mathrm{veh} / \mathrm{km} / \text { lane, } \rho_{0} \in\left[0, \rho_{\max }\right] \text {, }
$$

where $\rho_{\max }=143 \mathrm{veh} / \mathrm{km} / \mathrm{lane}$ is given by the PW model. Note that the critical density given by the PW fundamental diagram is $\rho_{\mathrm{c}}=30 \mathrm{veh} / \mathrm{km} / \mathrm{lane}$ and that the characteristic velocity is

Table 2

Parameters for the PW model

\begin{tabular}{lll}
\hline$\tau(\mathrm{s})$ & $\mu=\sqrt{-V_{\mathrm{e}}^{\prime}(\rho) / 2 \tau}(\mathrm{km} / \mathrm{h})$ & $V_{\mathrm{e}}(\rho)(\mathrm{veh} / \mathrm{km} / \mathrm{lane})$ \\
\hline 25 & 56 & $V_{\mathrm{e}}=\min \left\{88.5,88.5\left[1.94-6\left(\frac{\rho}{143}\right)+8\left(\frac{\rho}{143}\right)^{2}-3.93\left(\frac{\rho}{143}\right)^{3}\right]\right\}$ \\
\hline
\end{tabular}



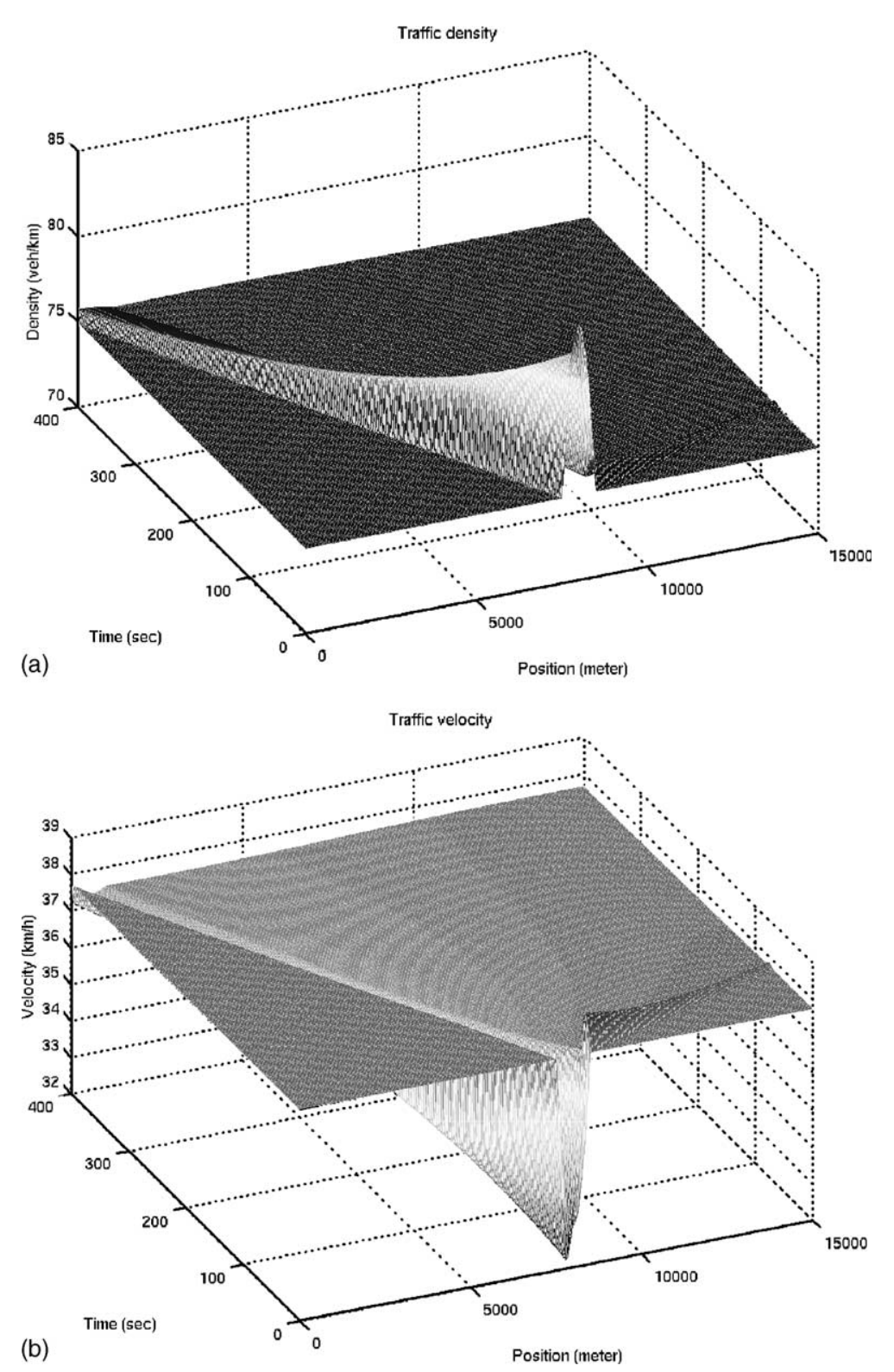

Fig. 3. Computed results for PW model (a) density $\rho$; (b) velocity $v$.

$v_{\mathrm{c}}=v_{0} \pm \mu$ and $\mu=56 \mathrm{~km} / \mathrm{h}$ (equivalently $\rho=51.5 \mathrm{veh} / \mathrm{km} / \mathrm{lane}$ ). In order to guarantee that the perturbation waves propagate backwards, the stability region for the equilibrium density is given by

$$
\rho_{\mathrm{c} 1}=52.4 \leqslant \rho_{0} \leqslant \rho_{\mathrm{c} 2}=114.7 \mathrm{veh} / \mathrm{km} / \text { lane }, \quad \rho_{0} \in\left[0, \rho_{\max }\right] .
$$

We design the following steady-state density profile as an initial condition to test the above results. 
density
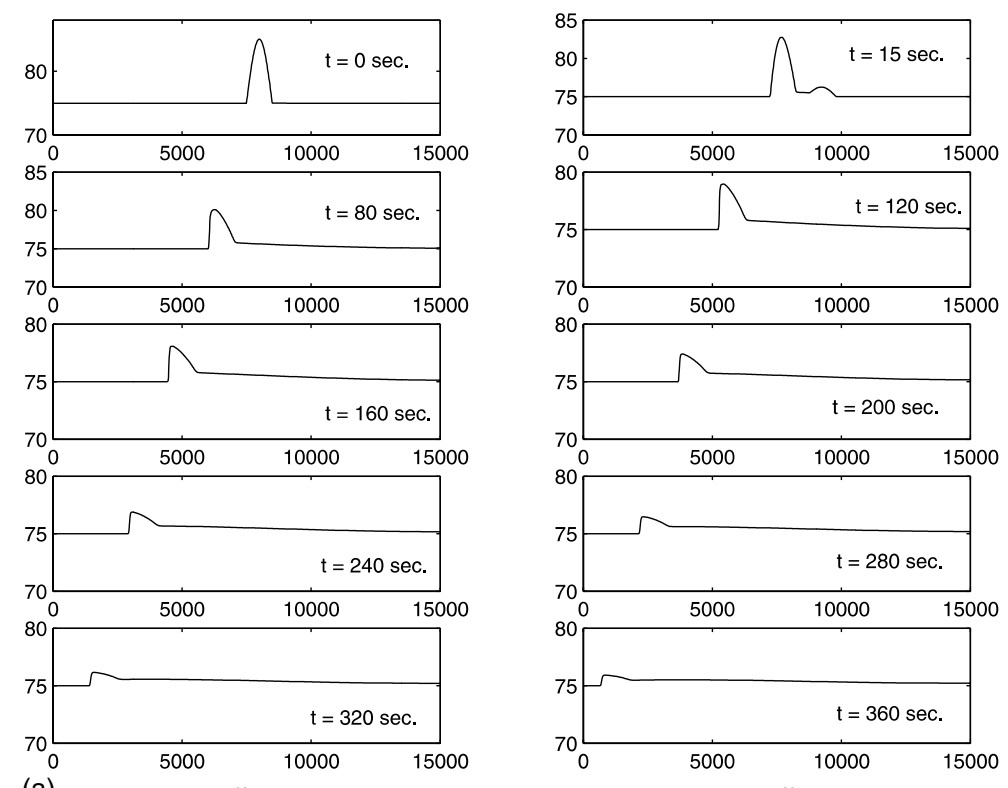

(a)
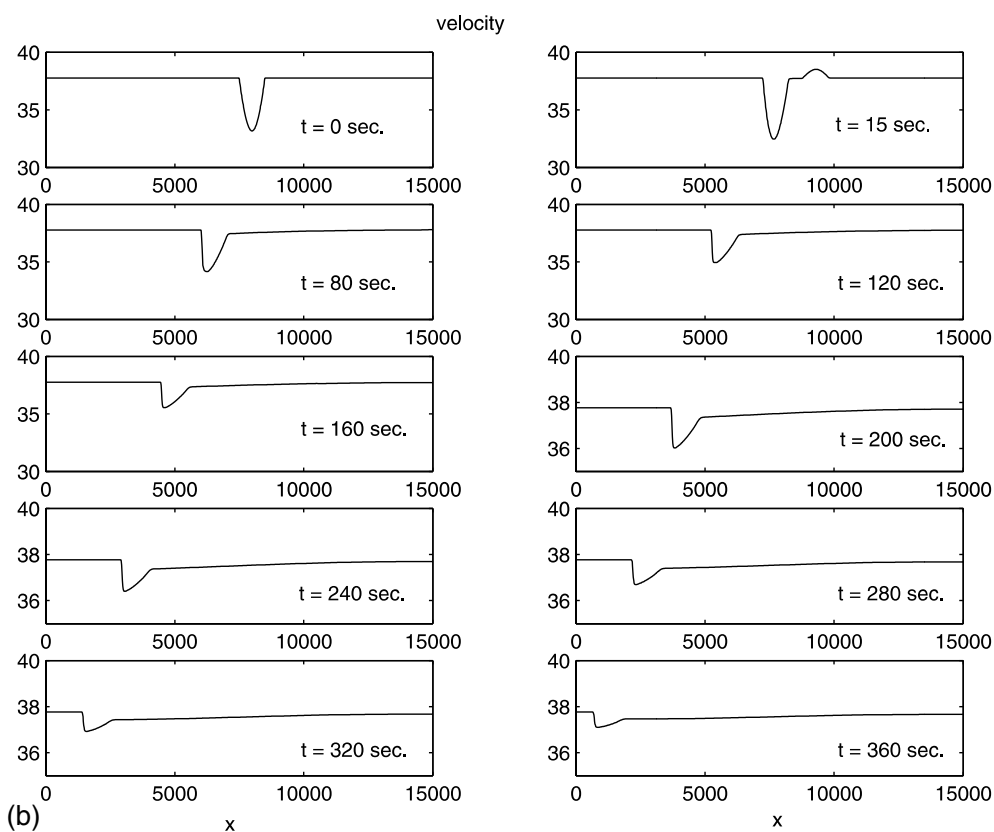

Fig. 4. Time snapshot results for PW model (a) density $\rho$; (b) velocity $v$.

$$
\rho(x, 0)= \begin{cases}\rho_{0}+\Delta \rho \cos \left(\frac{2 \pi\left(x-x_{0}\right)}{4 \Delta x}\right) & x_{0}-\Delta x \leqslant x \leqslant x_{0}-\Delta x \\ \rho_{0} & \text { other } x \in[0, L]\end{cases}
$$



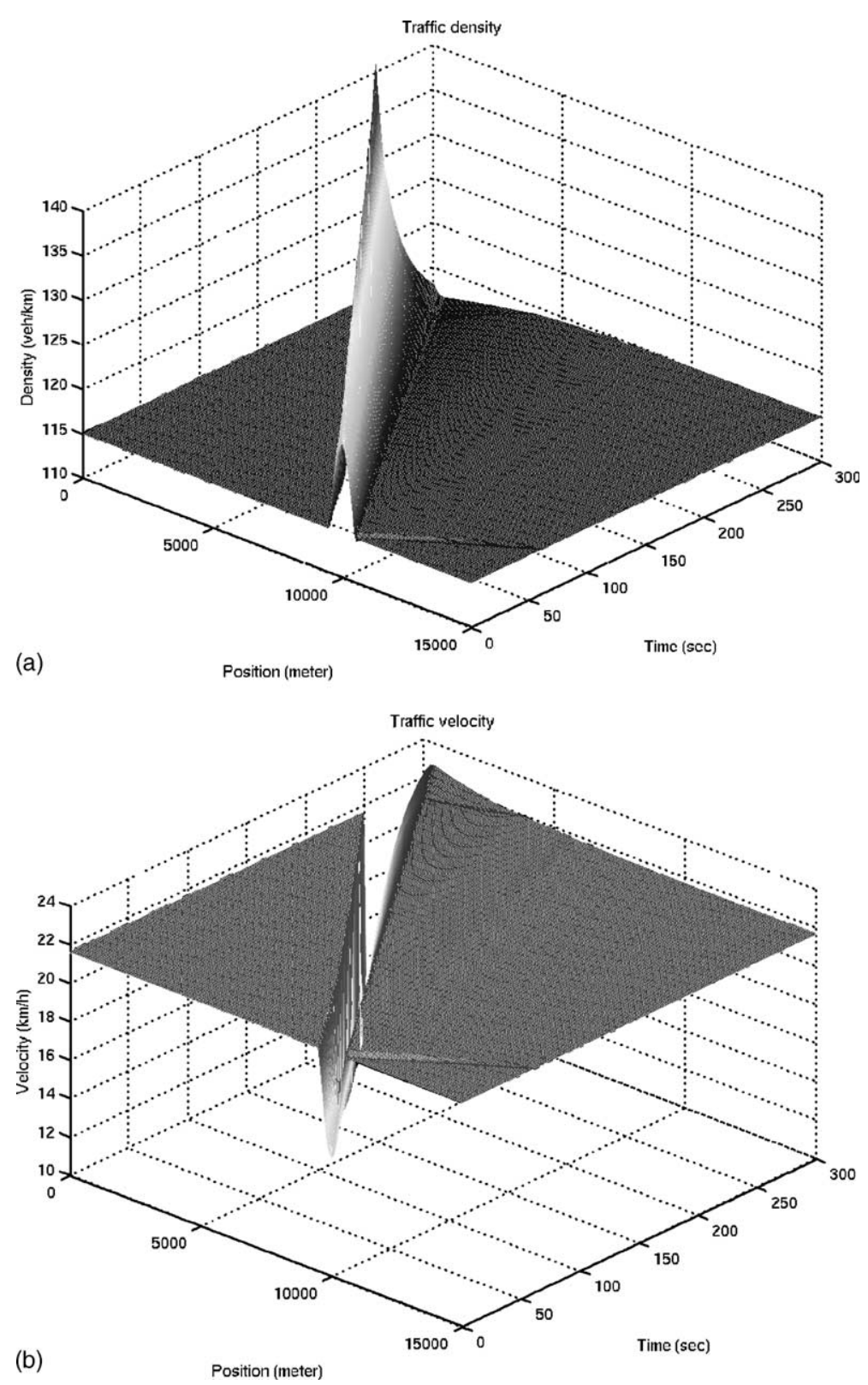

Fig. 5. Computed results for PW model (unstable) (a) density $\rho$; (b) velocity $v$.

where $L=15000 \mathrm{~m}$ is the length of the highway. In our computations, we assume that the highway is long enough so that we do not need to feed the specific boundary conditions, and zero order extrapolation is used instead. ${ }^{9}$

\footnotetext{
${ }^{9}$ We can apply other boundary conditions. However, in this paper, we only test the stability criterion.
} 

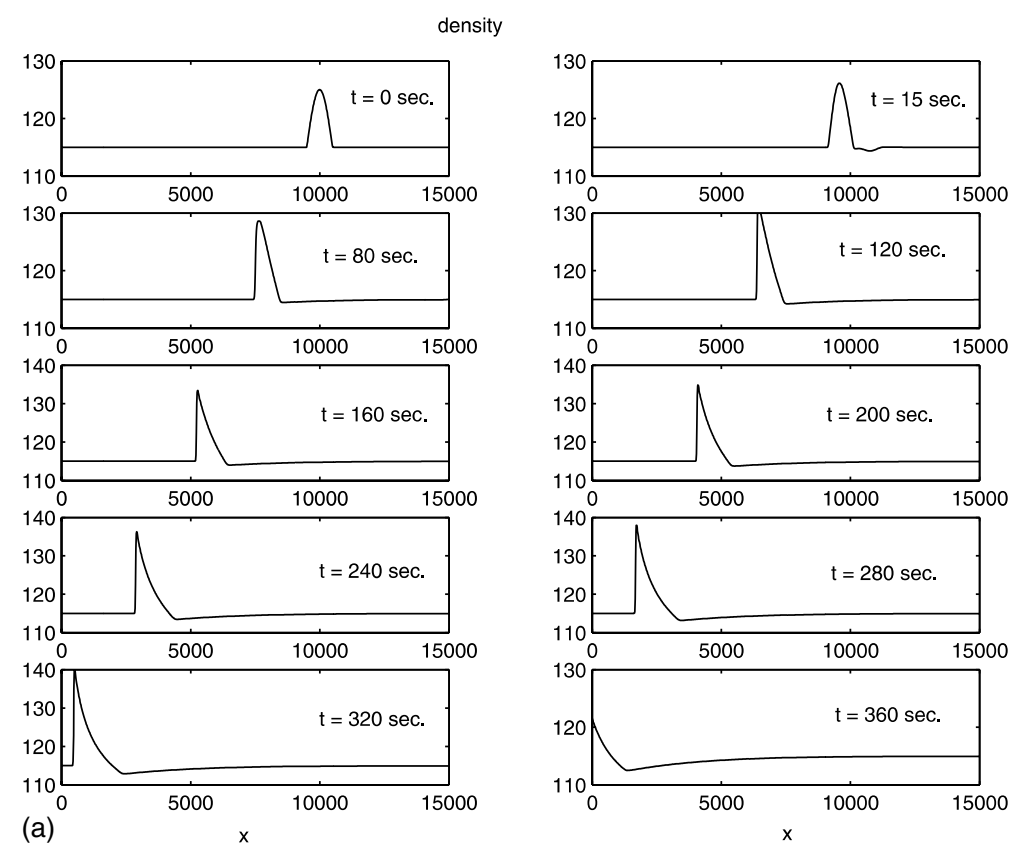

velocity
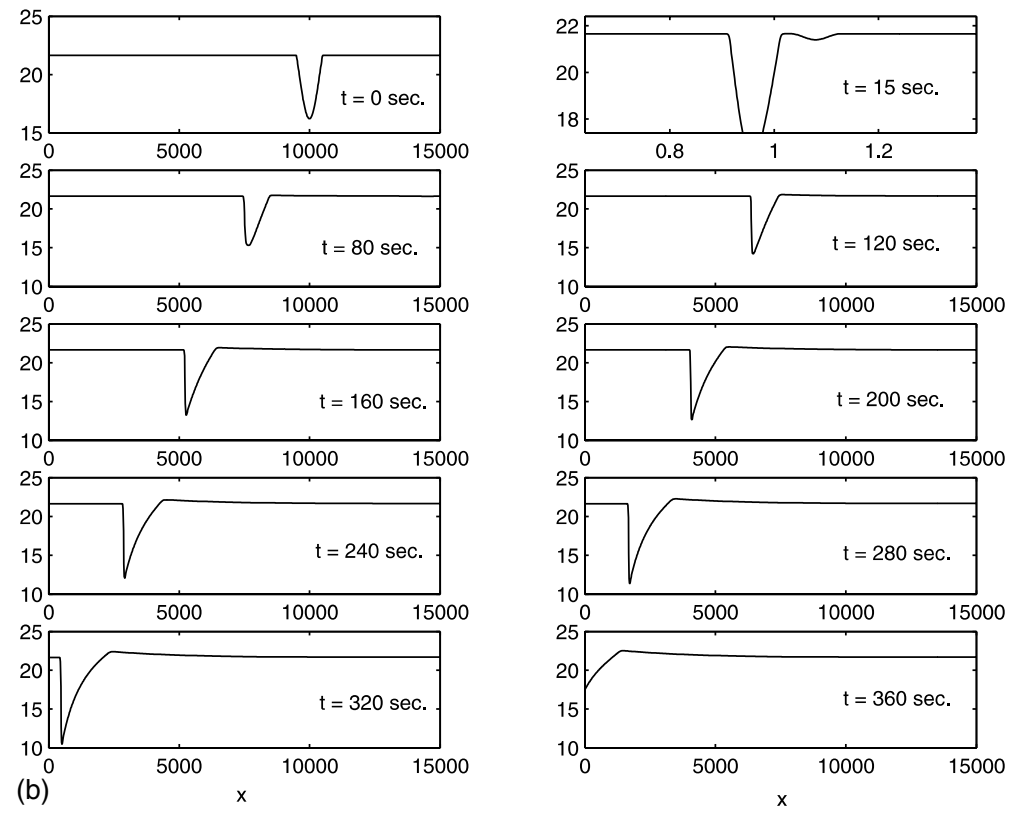

Fig. 6. Time snapshot results for PW model (unstable) (a) density $\rho$; (b) velocity $v$.

The first scenario is a simulation of a stable situation. We choose the following initial conditions in Eq. (26): 


$$
\rho_{0}=75 \mathrm{veh} / \mathrm{km} / \text { lane }, \quad \Delta \rho=10 \mathrm{veh} / \mathrm{km} / \text { lane }, \quad x_{0}=10000 \mathrm{~m}, \quad \Delta x=500 \mathrm{~m}
$$

and the numerical results are given in Figs. 3 and 4.

The second scenario is a simulation of an unstable situation. We choose the following initial conditions in Eq. (26):

$$
\rho_{0}=115 \mathrm{veh} / \mathrm{km} / \text { lane }, \quad \Delta \rho=10 \mathrm{veh} / \mathrm{km} / \text { lane }, \quad x_{0}=10000 \mathrm{~m}, \quad \Delta x=500 \mathrm{~m}
$$

and the numerical results are given in Figs. 5 and 6.

From above two examples, we observe that there are two perturbed waves generated by the initial conditions: one is moving forwards and the other backwards. The magnitude of the forward wave is much smaller than that of the backward wave. Moreover, the forward wave disappears quickly and the backward wave does not. For the first case, $\rho_{\mathrm{c} 1} \leqslant \rho_{0} \leqslant \rho_{\mathrm{c} 2}$, and by the analysis, both the backward and forward perturbed waves die out, so the perturbed waves become flat around $\rho_{0}$. However, for the second case, $\rho_{0}>\rho_{\mathrm{c} 2}$, the backward wave becomes divergent and it finally generates a shock wave, even though the forward wave dies out.

\section{Conclusions}

In this paper we discussed the traffic flow propagation stability using a nonlinear stability analysis technique. We first defined traffic flow stability by using macroscopic traffic flow variables. A generalized macroscopic traffic model was used for nonlinear stability analysis through the wavefront expansion method. A generalized stability criterion for macroscopic stability was proposed. For various existing second-order macroscopic models we calculated the stability conditions and compared them with stability conditions that were obtained through linearized analysis by previous authors. We found that the proposed stability analysis yields the same conditions as previously obtained results, in the case where such results existed. However, the nonlinear stability analysis in this paper gives more precise stability information along the perturbation propagation than previous approximate linear stability approaches. Moreover, stability conditions were obtained for nonlinear models, which were not previously analyzed using linearization techniques. A very interesting fact that was found in this study is that the downstream-moving propagation, which travels faster than traffic in second-order models and which, as pointed out by Daganzo (1995b), constitutes a major deficiency of most to second-order models, since it does not occur in actual systems, decays quickly. Some numerical examples have been presented to illustrate the theoretical results obtained in this paper. The numerical calculations are based on CLAWPACK, a computational package for conservation laws. The numerical results confirm the stability analysis for a PW model. The stability condition found in this paper can be further used for prediction of traffic stability and to perform a qualitative analysis of the relationships between traffic behaviors and traffic model parameters and structures.

\section{References}

Cremer, M., Papageorgiou, M., 1981. Parameter identification for a traffic flow model. Automatica 17 (6), 837-843.

Daganzo, C.F., 1994. The cell transmission model: a dynamic representation of highway traffic consistent with the hydrodynamic theory. Transportation Research B 28 (4), 269-287. 
Daganzo, C.F., 1995a. A finite difference approximation of the kinematic model of traffic flow. Transportation Research B 29 (4), 261-276.

Daganzo, C.F., 1995b. Requiem for second-order fluid approximations of traffic flow. Transportation Research B 29 (4), 277-286.

del Castillo, J.M., 2001. Propagation of perturbations in dense traffic flow: a model and its implications. Transportation Research B 35 (4), 367-389.

del Castillo, J.M., Pintado, P., Benítez, F.G., 1994. A formulation for the reaction time of traffic flow models. Transportation Research B 28 (1), 35-60.

Helbing, D., Treiber, M., 1999. Numerical simulation of macroscopic traffic equations. Computing in Science and Engineering 1 (5), 89-99.

Holland, E.N., 1998. A generalized stability criterion for motorway traffic. Transportation Research B 32 (2), 141-154.

Kerner, B.S., Konhäuser, P., 1993. Cluster effect in initially homogeneous traffic flow. Physical Review E 48 (4), 23352338.

Leo, C.J., Pretty, R.L., 1992. Numerical simulation of macroscopic continuum traffic models. Transportation Research B 26 (3), 207-220.

LeVeque, R.J., 1992. Numerical Methods for Conservation Laws, second ed. In: Lectures in Mathematics ETH. Birkhäuser Verlag, Basel, Switzerland.

LeVeque, R.J., 1997. Wave propagation algorithms for multidimensional hyperbolic systems. Journal of Computational Physics 131, 327-353.

LeVeque, R.J., 2000. CLAWPACK Version 4.0 User's Guide, University of Washington, also http://www.amath. washington.edu/ claw, Seattle, WA.

Lighthill, M.J., Whitham, J.B., 1955. On kinematic waves II: a theory of traffic flow on long crowded roads. Proceeding of the Royal Society A 229, 317-345.

Liu, G., Lyrintzis, A.S., Michalopoulos, P.G., 1997. Numerical simulation of freeway traffic flow. Journal of Transportation Engineering 123 (6), 503-513.

Liu, G., Lyrintzis, A.S., Michalopoulos, P.G., 1998. Improved high-order model for freeway traffic flow. Transportation Research Board 1644, 37-46.

Michalopoulos, P.G., Yi, P., Lyrintzis, A.S., 1993. Continuum modelling of traffic dynamics for congested freeways. Transportation Research B 27 (4), 315-332.

Papageorgiou, M., Blosseville, J.M., Hadj-Salem, H., 1989. Macroscopic modelling of traffic flow on the boulevard peripherique in Paris. Transportation Research B 23 (1), 29-47.

Payne, H.J., 1971. Models of freeway traffic and control. Simulation Council Preceding Series 1 (1), 51-61.

Payne, H.J., 1979. FREELO: a macroscopic simulation model of freeway traffic. Transportation Research Record 772 , $68-75$.

Phillips, W.F., 1979. A kinetic model for traffic flow with continuum implications. Transportation Planning and Technology 5, 131-138.

Richard, P.I., 1956. Shock waves on the highway. Operations Research 4, 42-51.

Swaroop, D., Rajagopal, K.R., 1999. Intelligent cruise control systems and traffic flow stability. Transportation Research C 7 (6), 329-352.

Treiber, M., Hennecke, A., Helbing, D., 1999. Derivation, properties and simulation of gas-kinetic-based nonlocal traffic model. Physical Review E 59 (1), 239-253.

Whitham, G.B., 1974. Linear and Nonlinear Waves. John Wiley and Sons Inc., New York, NY.

Zhang, H.M., 1998. A theory of nonequilibrium traffic flow. Transportation Research B 32 (7), 485-498.

Zhang, H.M., 1999. Analyses of the stability and wave properties of a new continuum traffic theory. Transportation Research B 33 (6), 399-415. 